\title{
A nonstandard linear finite element method for a planar elasticity problem
}

\author{
S. Barbeiro, J.A. Ferreira \\ Universidade de Coimbra, Faculdade de Ciências e Tecnologia, Departamento de Matemática, Apartado 3008, \\ 3000 Coimbra, Portugal
}

\begin{abstract}
The aim of this work is to present a nonstandard linear finite element method for a planar elasticity problem. The error for the solution computed with this method is estimated with respect to $H^{1} \times H^{1}$-norm and second-order convergence is shown. @ 2001 IMACS. Published by Elsevier Science B.V. All rights reserved.
\end{abstract}

\section{Introduction}

In most physical applications quantities are governed by systems of partial differential equations, not just by one equation. For instance, the deformations and stresses of elastic and inelastic bodies subject to load, studied in solid mechanics, are governed by systems of partial differential equations.

For the computation of a numerical approximation of the solution of a system of partial differential equations, finite element methods and finite difference methods are the numerical methods usually used.

In this paper we study a new linear finite element method for a planar elasticity problem which was, for the scalar case, presented by one of the authors in [7]. This method enables us to compute the numerical approximation to the displacement with an improved accuracy when compared with standard linear finite element methods described in the literature as for instance in $[1,3,12]$. This new method has two main features: on the one hand, it is based on a family of triangulations of the domain which does not need to be quasi-uniform and regular, and on the other hand, the finite element solution computed presents second order convergence with respect to $H^{1} \times H^{1}$-norm. This last property of the linear finite element method implies that the gradient of each component of the displacement is superconvergent.

About two decades ago, Zlámal [23] has already found superconvergence of the gradient for certain quadrature finite element solutions on nearly rectangular grids. Furthermore, Brandts [2] has found superconvergence of the gradient but there the grids were assumed regular and quasi-uniform.

Noting that the nonstandard finite element method studied in this work is equivalent to a carefully defined finite difference method, we conclude that this last method is supraconvergent. Supraconvergent

E-mail addresses: silvia@mat.uc.pt (S. Barbeiro), ferreira@mat.uc.pt (J.A. Ferreira). 
finite difference schemes have been largely studied in the literature and without being exhaustive we mention $[4,6-11,13,15,16,22]$.

The paper is organized as follows. In Section 2 we present the problem that we intend to solve. The nonstandard linear finite element method is described in Section 3. In Section 4 we present a finite difference method equivalent to the linear finite element method described in Section 3. The study of the $H^{1} \times H^{1}$-norm of the error is considered in Section 5. An example illustrating the performance of the method is considered in Section 6.

\section{The boundary value problem}

We begin with some notation. Let $v=\left(v_{1}, v_{2}\right)$ be a function of two variables. We define $\operatorname{div}(v)$ and $\operatorname{grad}(v)$ by

$$
\operatorname{div}(v)=\frac{\partial v_{1}}{\partial x}+\frac{\partial v_{2}}{\partial y}, \quad \operatorname{grad}(v)=\left[\begin{array}{ll}
\frac{\partial v_{1}}{\partial x} & \frac{\partial v_{1}}{\partial y} \\
\frac{\partial v_{2}}{\partial x} & \frac{\partial v_{2}}{\partial y}
\end{array}\right] .
$$

Let $A=\left[a_{i j}\right]_{i, j=1,2}$ be a matrix with $a_{i j}, i, j=1,2$, functions of two variables. By $\operatorname{div}(A)$ we denote the following function:

$$
\operatorname{div}(A)=\left[\begin{array}{l}
\frac{\partial a_{11}}{\partial x}+\frac{\partial a_{12}}{\partial y} \\
\frac{\partial a_{21}}{\partial x}+\frac{\partial a_{22}}{\partial y}
\end{array}\right]
$$

and we consider in the space of real two-by-two matrices the following inner product:

$$
A: B=\sum_{i, j=1}^{2} a_{i j} b_{i j},
$$

where $A=\left[a_{i j}\right], B=\left[b_{i j}\right]$. By $\operatorname{tr}(A)$ we denote the trace of the matrix $A$.

Let us define now the boundary value problem that we consider in this work. By $\Omega \subset \mathbb{R}^{2}$ we denote an union of rectangles and by $\partial \Omega$ we represent its boundary. We consider an isotropic material in the configuration space $\Omega$ and a body force $f$. By the static theory of linear elasticity, the displacement $u$ is the solution of the following system of partial differential equations

$$
-\operatorname{div}(\sigma(u))=f \quad \text { in } \Omega,
$$

with the displacement boundary condition

$$
u=g \quad \text { on } \partial \Omega \text {. }
$$

In (3), $\sigma(u)$ denotes the stress tensor defined by

$$
\sigma(u)=2 \mu \varepsilon(u)+\lambda \operatorname{tr}(\varepsilon(u)) I_{2},
$$

where $I_{2}$ is the identity two-by-two matrix, and

$$
\varepsilon(u)=\frac{1}{2}\left(\operatorname{grad}(u)+\operatorname{grad}(u)^{t}\right) .
$$

By $\mu, \lambda$ we represent the Lamé constants. 
We define in what follows a variational problem for the pure displacement problem (3) and (4). In $\boldsymbol{H}=H^{1}(\Omega) \times H^{1}(\Omega)$ and $\boldsymbol{L}^{2}=L^{2}(\Omega) \times L^{2}(\Omega)$ we consider the inner products

$$
(u, v)_{\boldsymbol{H} \times \boldsymbol{H}}=\left(u_{1}, v_{1}\right)_{H^{1}(\Omega)}+\left(u_{2}, v_{2}\right)_{H^{1}(\Omega)},
$$

for $u, v \in \boldsymbol{H}$, and

$$
(u, v)_{L^{2}}=\left(u_{1}, v_{1}\right)_{L^{2}(\Omega)}+\left(u_{2}, v_{2}\right)_{L^{2}(\Omega)}
$$

for $u, v \in \boldsymbol{L}^{2}$.

Let $a(\cdot, \cdot)$ be the sesquilinear form

$$
a(u, v)=\int_{\Omega}(2 \mu \varepsilon(u): \varepsilon(v)+\lambda \operatorname{div}(u) \operatorname{div}(v)) \mathrm{d} x \mathrm{~d} y
$$

for $(u, v) \in \boldsymbol{H} \times \boldsymbol{H}$. Considering $a(\cdot, \cdot)$ we introduce the variational problem:

Find $u \in \boldsymbol{H}$ such that

$$
u=g \quad \text { on } \partial \Omega \quad \text { and } \quad a(u, v)=(f, v)_{L^{2}(\Omega)}, \quad \forall v \in \boldsymbol{H}_{0},
$$

where $\boldsymbol{H}_{0}=H_{0}^{1}(\Omega) \times H_{0}^{1}(\Omega)$.

It is known that if $w \in \boldsymbol{H}$ is such that $\left.w\right|_{\partial \Omega}=g$, and if $u^{*} \in \boldsymbol{H}_{0}$ is solution of the variational problem

$$
a\left(u^{*}, v\right)=(f, v)_{L^{2}(\Omega)}-a(w, v), \quad \forall v \in \boldsymbol{H}_{0},
$$

then $u=u^{*}-w$ is solution of (5).

Attending to this that we consider in what follows homogeneous Dirichlet boundary conditions $(g=0)$.

The first Korn inequality enables us to conclude the ellipticity of $a(\cdot, \cdot)$ in $\boldsymbol{H}_{0} \times \boldsymbol{H}_{0}$ and so the next result:

Theorem 1. If $f \in \boldsymbol{H}^{-1}$ then exists a unique $u \in \boldsymbol{H}_{0}$ satisfying (5).

\section{The finite element method}

In this section we define the discrete variational problem (9) which enables us to compute an approximation to the solution of the variational problem (5) with $g=0$.

The finite element method that we consider is based on two specials triangulations which are induced by a nonuniform rectangular grid

$$
\mathbb{R}_{H}=\mathbb{R}_{1} \times \mathbb{R}_{2} \subset \mathbb{R}^{2},
$$

where $h=\left(h_{j}\right)_{\mathbb{Z}}$ and $k=\left(k_{\ell}\right)_{\mathbb{Z}}$ are sequences of positive numbers,

$$
\mathbb{R}_{1}=\left\{x_{j} \in \mathbb{R}: x_{j+1}=x_{j}+h_{j}, j \in \mathbb{Z}\right\}
$$

with $x_{0} \in \mathbb{R}$ given, and $\mathbb{R}_{2}$ is defined analogously to $\mathbb{R}_{1}$ with the meshsize vector $k$ in place of $h$.

Let $\Omega_{H}, \partial \Omega_{H}$ and $\bar{\Omega}_{H}$ be the intersection of $\mathbb{R}_{H}$ with $\Omega, \partial \Omega$ and $\bar{\Omega}$, respectively, that is,

$$
\Omega_{H}=\Omega \cap \mathbb{R}_{H}, \quad \partial \Omega_{H}=\partial \Omega \cap \mathbb{R}_{H}, \quad \bar{\Omega}_{H}=\bar{\Omega} \cap \mathbb{R}_{H} .
$$


The grid $\bar{\Omega}_{H}$ is assumed to satisfy the following condition with respect to the region $\Omega$.

(Reg) Let $\square$ be any rectangle $\left(x_{j}, x_{j+1}\right) \times\left(y_{\ell}, y_{\ell+1}\right)$ formed by the grid $\mathbb{R}_{H}$. Then $\square \cap \partial \Omega$ is empty.

The triangulations that we consider are related to the grid $\bar{\Omega}_{H}$, which we call $\mathcal{T}_{H}^{(1)}$ and $\mathcal{T}_{H}^{(2)}$. They are obtained from the disjoint decomposition

$$
\mathbb{R}_{H}=\mathbb{R}_{H}^{(1)} \dot{\cup} \mathbb{R}_{H}^{(2)},
$$

where the sums $j+\ell$ of the indices of the points $\left(x_{j}, y_{\ell}\right)$ in $\mathbb{R}_{H}^{(1)}$ and in $\mathbb{R}_{H}^{(2)}$ is even or odd, respectively. To simplify the following definition we introduce $\mathbb{R}_{H}^{(3)}=\mathbb{R}_{H}^{(1)}$. With each point $\left(x_{j}, y_{\ell}\right) \in \mathbb{R}_{H}$ we associate the triangles $\Delta_{j, \ell}^{(i)}, i=1,2,3,4$, that have a right angle at $\left(x_{j}, y_{\ell}\right)$ and two of the four closest neighbor grid points of $\left(x_{j}, y_{\ell}\right)$ as further vertices. We then define the triangulations

$$
\mathcal{T}_{H}^{(s)}=\left\{\Delta_{j, \ell}^{(i)} \subset \bar{\Omega},\left(x_{j}, y_{\ell}\right) \in \mathbb{R}_{H}^{(s)}, i \in\{1,2,3,4\}\right\}, \quad s=1,2,
$$

of $\bar{\Omega}(\stackrel{\circ}{\Delta}$ denotes the interior of $\Delta)$. Fig. 1 shows an example of one of these triangulations.

By $W_{H}$ we denote the space of grid functions defined on $\bar{\Omega}_{H}$ and by $\stackrel{\circ}{W}_{H}$ we represent the subspace of $W_{H}$ of grid functions vanishing on the boundary grid points $\partial \Omega_{H}$. Let $\stackrel{\circ}{W}_{H}$ be the set $\stackrel{\circ}{W}_{H} \times \stackrel{\circ}{W}_{H}$.

Let $\mathcal{T}_{H}$ be any triangulation of $\bar{\Omega}$ such that the nodes of $\mathcal{T}_{H}$ coincide with $\bar{\Omega}_{H}$. By $P_{H} v_{H}$ we denote $\left(P_{H} v_{H, 1}, P_{H} v_{H, 2}\right)$ where $v_{H}=\left(v_{H, 1}, v_{H, 2}\right) \in \stackrel{\circ}{W}_{H}$ and $P_{H} v_{H, i}$ is the continuous piecewise linear interpolation of $v_{H, i}(i=1,2)$ with respect to $\mathcal{T}_{H}$.

In what follows we define a discrete variational problem which enables us to compute the numerical approximation to the solution of (5) with $g=0$. Let $a_{H}(\cdot, \cdot)$ be the sesquilinear form defined by

$$
a_{H}\left(w_{H}, v_{H}\right)=\frac{1}{2}\left(a_{H}^{(1)}\left(w_{H}, v_{H}\right)+a_{H}^{(2)}\left(w_{H}, v_{H}\right)\right)
$$

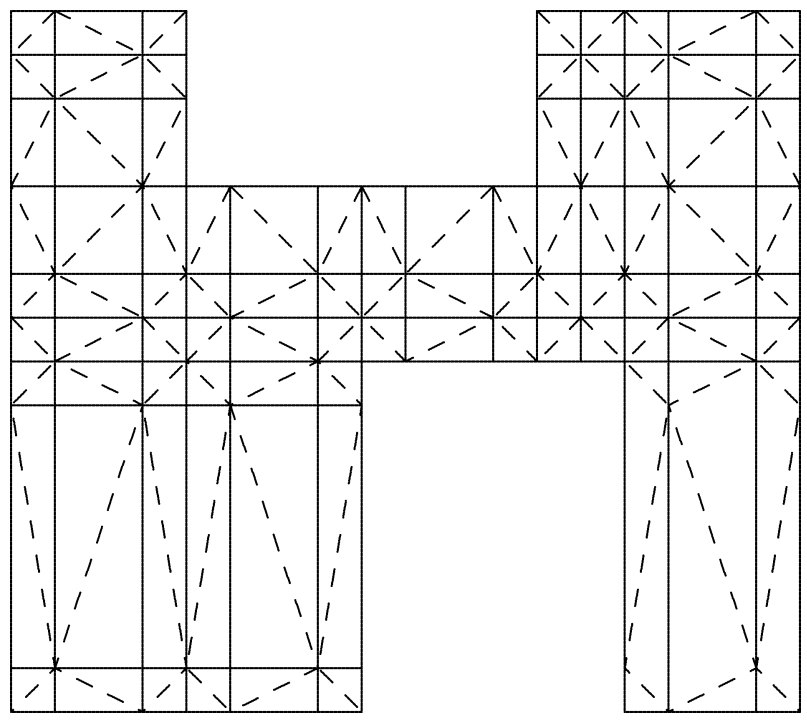

Fig. 1. Triangulation $\mathcal{T}_{H}^{(s)}$. 
for $w_{H}, v_{H} \in \stackrel{\circ}{\boldsymbol{W}}_{H} \times \stackrel{\circ}{\boldsymbol{W}}_{H}$, with $a_{H}^{(s)}\left(w_{H}, v_{H}\right)$, defined by

$$
a_{H}^{(s)}\left(w_{H}, v_{H}\right)=\sum_{\Delta \in \mathcal{T}_{H}^{(s)}} \int_{\Delta}\left(2 \mu \varepsilon\left(P_{H}^{(s)} w_{H}\right): \varepsilon\left(P_{H}^{(s)} v_{H}\right)+\lambda \operatorname{div}\left(P_{H}^{(s)} w_{H}\right) \operatorname{div}\left(P_{H}^{(s)} v_{H}\right)\right) \mathrm{d} x \mathrm{~d} y,
$$

for $s=1,2$.

Then our discrete variational problem is:

Find $u_{H} \in \stackrel{\circ}{\boldsymbol{W}}_{H}$ such that

$$
a_{H}\left(u_{H}, v_{H}\right)=\left(R_{H} f, v_{H}\right)_{H}, \quad \forall v_{H} \in \stackrel{\circ}{\boldsymbol{W}}_{H},
$$

where $R_{H}$ denotes the pointwise restriction operator.

In $(9),(\cdot, \cdot)_{H}$ represents the inner product

$$
\left(w_{H}, v_{H}\right)_{H}=\sum_{\left(x_{i}, y_{j}\right) \in \Omega_{H}} \omega_{i j}\left(w_{1, i j} v_{1, i j}+w_{2, i j} v_{2, i j}\right)
$$

with

$$
\omega_{i j}=\frac{h_{i}+h_{i+1}}{2} \frac{k_{j}+k_{j+1}}{2} .
$$

In what follows we rewrite $a_{H}^{(s)}(\cdot, \cdot)$ in an equivalent form. In order to do that we consider the sesquilinear forms

$$
\begin{aligned}
& a_{x x}^{(s)}\left(w_{H}, v_{H}\right)=\sum_{\Delta \in \mathcal{T}_{H}^{(s)}} \int_{\Delta}\left(P_{H}^{(s)} w_{H}\right)_{x}\left(P_{H}^{(s)} v_{H}\right)_{x} \mathrm{~d} x \mathrm{~d} y, \\
& a_{y y}^{(s)}\left(w_{H}, v_{H}\right)=\sum_{\Delta \in \mathcal{T}_{H}^{(s)}} \int_{\Delta}\left(P_{H}^{(s)} w_{H}\right)_{y}\left(P_{H}^{(s)} v_{H}\right)_{y} \mathrm{~d} x \mathrm{~d} y
\end{aligned}
$$

and

$$
a_{x y}^{(s)}\left(w_{H}, v_{H}\right)=\sum_{\Delta \in \mathcal{T}_{H}^{(s)}} \int_{\Delta}\left(P_{H}^{(s)} w_{H}\right)_{x}\left(P_{H}^{(s)} v_{H}\right)_{y} \mathrm{~d} x \mathrm{~d} y
$$

for $w_{H}, v_{H} \in \stackrel{\circ}{W}_{H}$. We define $a_{y x}^{(s)}(\cdot, \cdot)$ as $a_{x y}^{(s)}(\cdot, \cdot)$ changing the position of the variables $x$ and $y$.

Using the sesquilinear forms $a_{x x}^{(s)}(\cdot, \cdot), a_{y y}^{(s)}(\cdot, \cdot), a_{x y}^{(s)}(\cdot, \cdot)$ and $a_{y x}^{(s)}(\cdot, \cdot)$, is easy to show that

$$
\begin{aligned}
a_{H}^{(s)}\left(w_{H}, v_{H}\right)= & (2 \mu+\lambda)\left(a_{x x}^{(s)}\left(w_{H, 1}, v_{H, 1}\right)+a_{y y}^{(s)}\left(w_{H, 2}, v_{H, 2}\right)\right) \\
& +\mu\left(a_{x x}^{(s)}\left(w_{H, 2}, v_{H, 2}\right)+a_{y y}^{(s)}\left(w_{H, 1}, v_{H, 1}\right)\right) \\
& +\mu\left(a_{x y}^{(s)}\left(w_{H, 2}, v_{H, 1}\right)+a_{y x}^{(s)}\left(w_{H, 1}, v_{H, 2}\right)\right) \\
& +\lambda\left(a_{y x}^{(s)}\left(w_{H, 2}, v_{H, 1}\right)+a_{x y}^{(s)}\left(w_{H, 1}, v_{H, 2}\right)\right)
\end{aligned}
$$

for $w_{H}, v_{H} \in \stackrel{\circ}{\boldsymbol{W}}_{H}$. 
The following stability result is consequence of the definition of $a_{H}(\cdot, \cdot)$.

Theorem 2. Exists a positive constant $C$ such that

$$
\left\|P_{H} v_{H}\right\|_{1} \leqslant C \sup _{\substack{0 \neq w_{H} \in \dot{W}_{H}\\}} \frac{\left|a_{H}\left(v_{H}, w_{H}\right)\right|}{\left\|P_{H} w_{H}\right\|_{1}}
$$

for all $v_{H} \in \stackrel{\circ}{W}_{H}$.

The proof of this theorem follows the steps of the proof of Theorem 2 of [7].

\section{An equivalent finite difference method}

In this section we define a finite difference method "equivalent" to the discrete variational problem (9) that is useful to implement the described finite element method.

For each grid point $\left(x_{j}, y_{\ell}\right) \in \mathbb{R}_{H}$ we define the central finite difference quotients

$$
\begin{aligned}
& \delta_{x}^{(1 / 2)} w_{j, \ell}=\frac{w_{j+1 / 2, \ell}-w_{j-1 / 2, \ell}}{x_{j+1 / 2}-x_{j-1 / 2}}, \quad \delta_{x}^{(1 / 2)} w_{j+1 / 2, \ell}=\frac{w_{j+1, \ell}-w_{j, \ell}}{x_{j+1}-x_{j}}, \\
& \delta_{x} w_{j, \ell}=\frac{w_{j+1, \ell}-w_{j-1, \ell}}{x_{j+1}-x_{j-1}},
\end{aligned}
$$

where $x_{j+1 / 2}=x_{j}+h_{j} / 2, x_{j-1 / 2}=x_{j}-h_{j-1} / 2$. Correspondingly, the central finite difference quotients with respect to the variable $y$ are defined.

We introduce now the following finite difference problem:

Find $u_{H} \in \stackrel{\circ}{\boldsymbol{W}}_{H} \quad$ such that $\quad A_{H} u_{H}=f \quad$ in $\Omega_{H}$,

with

$$
A_{H} u_{H}=\left[\begin{array}{c}
(2 \mu+\lambda) \delta_{x}^{(1 / 2)} \delta_{x}^{(1 / 2)} u_{H, 1}+\mu \delta_{x} \delta_{y} u_{H, 2}+\lambda \delta_{y} \delta_{x} u_{H, 2}+\mu \delta_{y}^{(1 / 2)} \delta_{y}^{(1 / 2)} u_{H, 1} \\
(2 \mu+\lambda) \delta_{y}^{(1 / 2)} \delta_{y}^{(1 / 2)} u_{H, 2}+\lambda \delta_{y} \delta_{x} u_{H, 1}+\mu \delta_{x} \delta_{y} u_{H, 1}+\mu \delta_{x}^{(1 / 2)} \delta_{x}^{(1 / 2)} u_{H, 2}
\end{array}\right] .
$$

Attending to the definitions of $a_{H}(\cdot, \cdot)$ and $A_{H}$ is easy to show the next result:

Proposition 1. Let the sesquilinear form $a_{H}(\cdot, \cdot)$ be defined by (7). With $A_{H}$ defined by (15), the equality

$$
a_{H}\left(v_{H}, w_{H}\right)=\left(A_{H} v_{H}, w_{H}\right)_{H}
$$

holds for $w_{H}, v_{H} \in \stackrel{\circ}{W}_{H}$.

Paying attention to the last proposition and to the stability inequality (13) we conclude that $A_{H}$ has inverse and is stable in the following sense: exists a positive constant $C$ independent of $H$ such that

$$
\left\|P_{H} v_{H}\right\|_{1} \leqslant C \sup _{\substack{0 \neq w_{H} \in \dot{W}_{H}\\}} \frac{\left|\left(A_{H} v_{H}, w_{H}\right)_{H}\right|}{\left\|P_{H} w_{H}\right\|_{1}},
$$

for $v_{H} \in \stackrel{\circ}{\boldsymbol{W}}_{H}$. 


\section{Bounding the error}

We consider in what follows a sequence of grids $\mathbb{R}_{H}$ defined using a sequence $\Lambda$ of $H=(h, k)$ such that the maximal mesh-size $H_{\max }$ tends to zero. By $\|\cdot\|_{r, \infty, \Delta}$ we represent the standard norm in $W^{r, \infty}(\Omega)$ if the underlying region is the triangle $\Delta$.

The truncation error for the finite difference operator $A_{H}$ is on nonuniform grids pointwise of order one. Nevertheless, in what follows, we show that (14) is second order convergent, that is, $\left\|P_{H} R_{H} u-P_{H} u_{H}\right\|_{1}=\mathrm{O}\left(H_{\max }^{2}\right)$ where $u_{H}$ and $u$ are respectively the finite difference solution (also finite element solution) and the solution of the elasticity problem.

Let us estimate now $\left\|P_{H} R_{H} u-P_{H} u_{H}\right\|_{1}$. Looking back at (13) we have

$$
\left\|P_{H} R_{H} u-P_{H} u_{H}\right\|_{1} \leqslant C \sup _{\substack{v_{H} \in W_{H} \\ v^{\prime}}} \frac{\left|a_{H}\left(R_{H} u, v_{H}\right)-\left(f, v_{H}\right)_{H}\right|}{\left\|P_{H} v_{H}\right\|_{1}}
$$

and an estimate to the error $\left\|P_{H} R_{H} u-P_{H} u_{H}\right\|_{1}$ is obtained estimating

$$
a_{H}\left(R_{H} u, v_{H}\right)-\left(R_{H} f, v_{H}\right)_{H}
$$

for $v_{H} \in \stackrel{\circ}{\boldsymbol{W}}_{H}$.

We observe that from [7] we have

$$
\begin{aligned}
& a_{x x}^{(s)}\left(R_{H} u_{i}, v_{H, i}\right)=-\left(R_{H} \frac{\partial^{2} u_{i}}{\partial x^{2}}, v_{H, i}\right)_{H}+R_{x x i}, \quad \text { for } i=1,2, \\
& a_{y y}^{(s)}\left(R_{H} u_{i}, v_{H, i}\right)=-\left(R_{H} \frac{\partial^{2} u_{i}}{\partial y^{2}}, v_{H, i}\right)_{H}+R_{y y i}, \quad \text { for } i=1,2,
\end{aligned}
$$

and

$$
a_{x y}^{(s)}\left(R_{H} u_{i}, v_{H, j}\right)=-\left(R_{H} \frac{\partial^{2} u_{i}}{\partial y \partial x}, v_{H, i}\right)_{H}+R_{x y i},
$$

for $i=1, j=2$ and $i=2, j=1$, where

$$
\left|R_{x x i}\right| \leqslant C\left(\sum_{\Delta \in \mathcal{T}_{H}^{(s)}}|\Delta| h_{\Delta}^{4}\left\|\frac{\partial^{3} u_{i}}{\partial x^{3}}\right\|_{1, \infty, \Delta}^{2}\right)^{1 / 2}\left\|P_{H} v_{H, i}\right\|_{1},
$$

where we have represented the area of the triangle $\Delta$ by $|\Delta|$. The bound for remainder term $R_{y y i}$ is obtained by taking $k_{\Delta}$ and $\partial / \partial y$ in place of $h_{\Delta}$ and $\partial / \partial x$ respectively in (22), and

$$
\left|R_{x y i}\right| \leqslant C\left(\sum_{\Delta \in \mathcal{T}_{H}^{(s)}}|\Delta|\left(h_{\Delta}^{4}+k_{\Delta}^{4}\right)\left(\left\|\frac{\partial^{3} u_{i}}{\partial^{2} y \partial x}\right\|_{1, \infty, \Delta}^{2}+\left\|\frac{\partial^{3} u_{i}}{\partial y \partial^{2} x}\right\|_{1, \infty, \Delta}^{2}\right)\right)^{1 / 2}\left\|P_{H} v_{H, j}\right\|_{1} .
$$

The bound for the remainder term $R_{y x i}$ is obtained by taking $\partial / \partial y$ in place of $\partial / \partial x$ in (23).

Altogether we have proved the following result:

Proposition 2. Let $u$ be in $C^{4}(\bar{\Omega}) \times C^{4}(\bar{\Omega})$. Then

$$
a_{H}\left(R_{H} u, v_{H}\right)=-\left(R_{H}[\operatorname{div}(\sigma(u))], v_{H}\right)_{H}+\tau\left(u, v_{H}\right)
$$


with

$$
\tau\left(u, v_{H}\right) \leqslant C\left(\sum_{\Delta \in \mathcal{T}_{H}}|\Delta|(\operatorname{diam} \Delta)^{4}\|u\|_{4, \infty, \Delta}^{2}\right)^{1 / 2}\left\|P_{H} v_{H}\right\|_{1},
$$

where $C$ is independent of the triangulation $\mathcal{T}_{H}$ and of $u$.

Finally, combining the last proposition and inequality (17) we conclude the main result of this work:

Theorem 3. If the solution of (3) and (4) is in $C^{4}(\bar{\Omega}) \times C^{4}(\bar{\Omega})$ with $g=0$, then the variational problem (9) and the finite difference problem (14) have a unique solution $u_{H}$ in $\stackrel{\circ}{W}_{H}$ satisfying the error estimate

$$
\left\|P_{H} R_{H} u-P_{H} u_{H}\right\|_{1} \leqslant C\left(\sum_{\Delta \in \mathcal{T}_{H}}|\Delta|(\operatorname{diam} \Delta)^{4}\|u\|_{4, \infty, \Delta}^{2}\right)^{1 / 2} .
$$

\section{Numerical example}

In the following example we show the performance of the method defined by (9) or equivalently the performance of the finite difference scheme (14).

Example 1. Let us consider the boundary value problem (3) defined on the rectangle $\Omega=(0,1) \times(0,1)$, with $\lambda=1, \mu=0.5$,

$$
f_{1}(x, y)=f_{2}(x, y)=\pi^{2}[-0.4 \cos (\pi(x+y))+0.1 \cos (\pi(x-y))]
$$

and $g=0$. This planar elasticity problem has the following solution:

$$
u_{1}(x, y)=u_{2}(x, y)=0.2 \sin (\pi x) \sin (\pi y) .
$$

We define the grid $\bar{\Omega}_{H, 1}$ taking $x_{0}=y_{0}=0$ and

$$
\begin{aligned}
& h_{j}=0.125, \quad j=1,2,13,14, \quad h_{j}=0.05, \quad j=3, \ldots, 12 \text {, } \\
& k_{\ell}=0.125, \quad \ell=1,2,13,14, \quad k_{\ell}=0.05, \quad \ell=3, \ldots, 12 \text {. }
\end{aligned}
$$

Introducing a new grid line between each grid line of $\bar{\Omega}_{H, 1}$ we obtain the grid $\bar{\Omega}_{H, 3}$. Following the last procedure we define the grids $\bar{\Omega}_{H, i}$ for $i=5,7$. Analogously we define the grids $\bar{\Omega}_{H, i}$ for $i=4,6,8$, using the same procedure where $\bar{\Omega}_{h, 2}$ is defined taking $x_{0}=y_{0}=0$ and

$$
\begin{array}{lll}
h_{j}=0.1, \quad j=1,2,15,16, & h_{j}=0.05, & j=3, \ldots, 14, \\
k_{\ell}=0.1, \quad \ell=1,2,15,16, & k_{\ell}=0.005, & \ell=3, \ldots, 14 .
\end{array}
$$

In Table 1 we present the number of points in the $x$ and $y$ directions which are denoted respectively by $N$ and $M$, the maximum step-size $H_{\max }$ and the norm $\|\cdot\|_{1}$ of the error. In Fig. 2 we plot the logarithm of the $\boldsymbol{H}$-norm of the error against the logarithm of the square of the maximum step-size that illustrates the convergence result.

From the values presented in last table we easily conclude that the average convergence rate is 1.95 which confirm the second order of convergence of the method stated in Theorem 3. 
Table 1

\begin{tabular}{lclc}
\hline Grid & Number of points & \multicolumn{1}{c}{$H_{\max }$} & $\left\|P_{H} R_{H} u-P_{H} u_{H}\right\|_{1}$ \\
\hline $\bar{\Omega}_{H, 1}$ & $N=M=14$ & 0.125 & 0.0167266 \\
$\bar{\Omega}_{H, 2}$ & $N=M=16$ & 0.1 & 0.00886546 \\
$\bar{\Omega}_{H, 3}$ & $N=M=28$ & 0.0625 & 0.00451434 \\
$\bar{\Omega}_{H, 4}$ & $N=M=32$ & 0.05 & 0.0023329 \\
$\bar{\Omega}_{H, 5}$ & $N=M=56$ & 0.03125 & 0.00116346 \\
$\bar{\Omega}_{H, 6}$ & $N=M=64$ & 0.025 & 0.00059468 \\
$\bar{\Omega}_{H, 7}$ & $N=M=112$ & 0.015625 & 0.000293906 \\
$\bar{\Omega}_{H, 8}$ & $N=M=128$ & 0.0125 & 0.000149632 \\
\hline
\end{tabular}

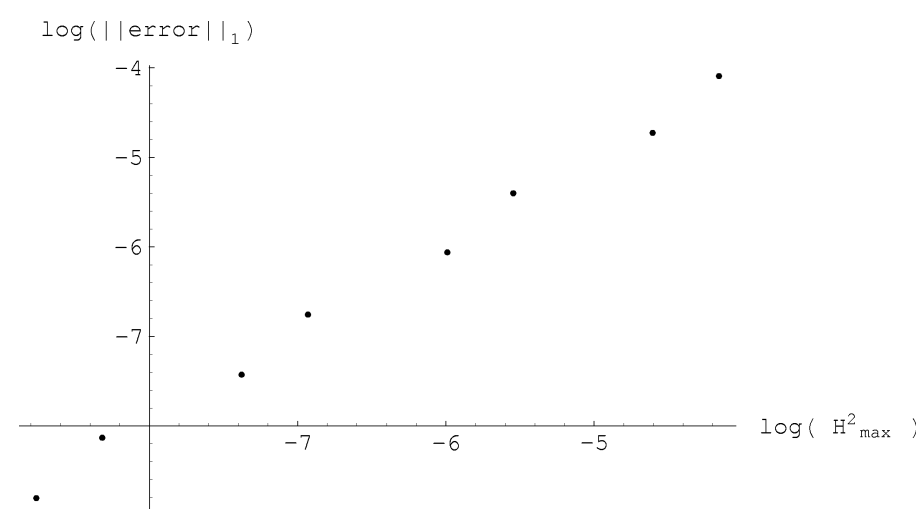

Fig. 2. The logarithm of the norm $\|\cdot\|_{1}$ of the error.

\section{Acknowledgements}

The authors gratefully acknowledge the support of this work by the Centro de Matemática da Universidade de Coimbra.

\section{References}

[1] D. Braess, Finite Elements: Theory, Fast Solvers, and Applications in Solid Mechanics, Cambridge University Press, 1997.

[2] J.H. Brandts, Superconvergence and a posteriori error estimation for triangular mixed finite elements, Numer. Math. 68 (1994) 331-324.

[3] S.C. Brenner, L.R. Scott, The Mathematical Theory of Finite Element Methods, Texts in Applied Mathematics, Springer, New York, 1994.

[4] F. de Hoog, D. Jackett, On the rate of convergence of finite difference schemes on nonuniform grids, J. Austral. Math Soc. Ser. B (1985) 247-256. 
[5] R. Duran, M.A. Muschietti, R. Rodrigues, On the asymptotic exactness of error estimates for linear triangular finite element methods, Numer. Math. 59 (1991) 107-127.

[6] J.A. Ferreira, The negative norms in the supraconvergence of FDM's for two-dimensional domains, Technical Report 97-06, 1997.

[7] J.A. Ferreira, R.D. Grigorieff, On the supraconvergence of elliptic finite difference schemes, Appl. Numer. Math. 28 (1998) 275-292.

[8] P.A. Forsyth, P.H. Sammon, Quadratic convergence for cell-centered grids, Appl. Numer. Math. 4 (1988) 377-394.

[9] B. Garcia-Archila, A supraconvergent scheme for the Korteweg-de Vries equation, Numer. Math. 61 (1992) 292-310.

[10] B. Garcia-Archila, J.M. Sanz-Serna, A finite difference formula for the discretization of $\mathrm{d}^{3} / \mathrm{d}^{3}$ on nonuniform grids, Math. Comp. 57 (1991) 239-257.

[11] R.D. Grigorieff, Some stability inequalities for compact finite difference operators, Math. Nach. 135 (1986) 93-101.

[12] W. Hackbusch, Theory and Numerical Treatment of Elliptic Differential Equations, Springer, Berlin, 1992.

[13] H.O. Kreiss, T.A. Manteuffel, B. Swartz, B. Wendroff, A.B. White Jr., Supraconvergent schemes on irregular grids, Math. Comp. 47 (1986) 537-554.

[14] P. Lesaint, M. Zlámal, Superconvergence of the gradient of finite element solutions, RAIRO Anal. Numér. 13 (1979) 139-166.

[15] C.D. Levermore, T.A. Manteuffel, A.B. White Jr., Numerical solutions of partial differential equations on irregular grids, in: Computational Techniques and Applications: CTAC-87, Sydney, 1987, North-Holland, Amsterdam/New York, 1987, pp. 417-426.

[16] T.A. Manteuffel, A.B. White Jr., The numerical solution of second order boundary value problems on nonuniform meshes, Math. Comp. 47 (1986) 511-535.

[17] M.A. Marletta, Supraconvergence of discretization methods on nonuniform meshes, M.Sc. Thesis, Oxford University, 1988.

[18] P. Monk, E. Süli, A convergence analysis of Yee's scheme on non-uniform grids, SIAM J. Numer. Anal. 28 (1994) 393-412.

[19] K.W. Morton, E.E. Süli, Evolution-Galerkin methods and their supraconvergence, Numer. Math. 71 (1995) 331-355.

[20] E. Süli, Convergence of finite volume schemes for Poisson's equation on non-uniform meshes, SIAM J. Numer. Anal. 28 (1991) 1419-1430.

[21] A. Weiser, M.F. Wheeler, On convergence of block-centered finite differences for elliptic problems, SIAM J. Numer. Anal. 25 (1988) 351-375.

[22] B. Wendroff, A.B. White Jr., Some supraconvergent schemes for hyperbolic equations on irregular grids, in: Nonlinear Hyperbolic Equations-Theory, Computation Methods, and Applications, Aachen, 1988, Notes on Numerical Fluid Mechanics, Vol. 24, Vieweg, Braunschweig, 1988, pp. 671-677.

[23] M. Zlámal, Superconvergence and reduced integration in finite element method, Math. Comp. 32 (1978) 663685. 